\title{
Indicadores de abastecimento de água e doenças de transmissão hídrica em municípios da Amazônia Oriental
}

\author{
Indicators of water supply and water transmission \\ diseases in Eastern Amazonian municipalities \\ Elizandra Perez Araújo' $\oplus^{\oplus}$, Helenilza Ferreira Albuquerque Cunha' ${ }^{\oplus}$,

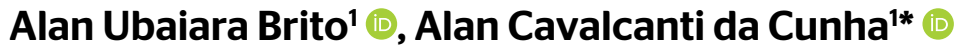

口-

\begin{abstract}
RESUMO
O objetivo da pesquisa foi correlacionar indicadores operacionais de abastecimento de água com a frequência de doenças de notificação compulsória de transmissão hídrica em seis municípios do estado do Amapá, Brasil. A metodologia consistiu no tratamento estatístico de dados de doenças de notificação compulsória de transmissão hídrica, disponibilizados pela Superintendência de Vigilância em Saúde do Amapá (período de 2007 a 2017), com indicadores operacionais de abastecimento de água consolidados pelo Sistema Nacional de Informações sobre Saneamento (período de 1996 a 2017). Os resultados mostraram, por meio de séries de regressões, que seis entre 20 indicadores operacionais apresentaram significância e correlação positiva com a ocorrência de doenças diarreicas agudas, independentemente do município $\left(R_{a j}{ }^{2}=0,75\right.$, $p<0,05)$, com tendência progressiva em relação à expansão dos serviços de saneamento básico. Conclui-se que os seis indicadores operacionais apresentaram correlação positiva com a ocorrência de doenças diarreicas agudas. Entretanto, apesar de ter ocorrido no período uma sensível expansão do sistema operacional de abastecimento de água, também houve aumento dessas doenças, sugerindo uma crescente tendência temporal do deficit de saneamento básico oferecido nesses municípios.
\end{abstract}

Palavras-chave: abastecimento de água; saúde pública; municípios amazônicos.

\begin{abstract}
The objective of the research was to correlate operational water supply indicators with the frequency of diseases with compulsory notification of water transmission in six municipalities in the state of Amapá/Brazil. The methodology consisted of the statistical treatment of data on diseases with compulsory notification of water transmission, made available by the Health Superintendence of Surveillance of Amapá (Superintendência de Vigilância em Saúde do Amapá) (period from 2007 to 2017), with operational water supply indicators consolidated by the National Sanitation Information System (Sistema Nacional de Informações sobre Saneamento) (period from 1996 to 2017). The results showed, through a series of regressions, that six out of twenty operational indicators, presented significance and positive correlation with acute diarrheal diseases, regardless of the municipality $\left(R_{a j}{ }^{2}=0.75, p<0.05\right)$, with a progressive trend of these diseases with the expansion of basic sanitation services. We concluded that the six operational indicators showed a positive correlation with acute diarrheal diseases. However, despite the expansion of the operating system of water supply in the period, there was also an increase in these diseases, suggesting a growing temporal trend of the basic sanitation deficit offered in these municipalities.
\end{abstract}

Keywords: water supply; public health; Amazonian municipalities.

\section{INTRODUÇÃO}

O estado do Amapá possui um dos piores índices de saneamento básico do Brasil (SNIS, 2019). Historicamente, mesmo na capital Macapá, têm sido mantidos baixos índices de saneamento, inclusive na dimensão mais onerosa do setor, o abastecimento de água (CUNHA et al., 2004; CUNHA et al., 2005). Esse problema decorre da poluição e do comprometimento de mananciais (OLIVEIRA et al., 2019), com potenciais reflexos sobre doenças de notificação compulsória de transmissão hídrica - NCTH (VIANA, FREITAS \& GIATTI, 2016).

Assim, o quadro atual do saneamento básico no estado do Amapá não somente é um reflexo da histórica falta de planejamento e investimento nesse

'Universidade Federal do Amapá - Macapá (AP), Brasil.

*Autor correspondente: alancunha12@gmail.com

Conflitos de interesse: os autores declaram não haver conflitos de interesse.

Financiamento: Conselho Nacional de Desenvolvimento Científico e Tecnológico (CNPq) \# 309684/2018-8, DPq/PROPESPg/UNIFAP.

Recebido: 15/05/2O2O - Aceito: 30/11 /2O2O - Reg. ABES: 20200179 
setor (SNIS, 2019), como também da profunda deterioração e abandono da infraestrutura em relação às sedes municipais localizadas em áreas rurais ou do interior do estado (UNIFAP, 2018).

Embora a importância do abastecimento de água esteja notoriamente associada com os aspectos sanitários e econômicos, é evidente a dificuldade do poder público em fornecer água potável em quantidade e qualidade suficiente e adequada à população urbana e rural dos pequenos municípios brasileiros (TSUTIYA, 2006). Por exemplo, a Lei $n^{\circ} 11.445 / 07$ estabelece as diretrizes para a política nacional de saneamento e entre suas competências, destaca-se a universalização do sistema de abastecimento de água potável, quantificada por uma série de indicadores de saneamento e saúde pública. Nesse caso, refere-se a todos terem o direito e o acesso ao serviço público de saneamento básico de boa qualidade (BRASIL, 2007).

Por esses motivos, os financiadores e gestores do setor têm se guiado por indicadores, com o objetivo de compreender como os planos, os programas, as ações e os projetos impactam outros indicadores, como os de saúde pública, buscando um bem maior, a universalização dos serviços de saneamento básico (KERRY, DAVID \& JOHN, 2012).

Entretanto, as entidades reguladoras de serviços de abastecimento de água têm como desafio a formulação e a implementação de um conjunto de resultados que impactem sobre os indicadores e permitam avaliar e gerenciar operacionalmente a eficiência da produção e a perda de água nos sistemas de abastecimento, por exemplo, sem os quais não seria possível avaliar o desempenho dos prestadores de serviços (BIASUTTI \& COELHO, 2019).

Quanto aos problemas de saúde pública, a notificação compulsória das doenças de transmissão hídrica, como os casos de doenças diarreicas agudas, é realizada diante da suspeita ou confirmação de doença ou agravo, mediante o atendimento de normas técnicas estabelecidas pela Secretaria de Vigilância em Saúde do Ministério da Saúde (SVS/MS). A comunicação de doenças à autoridade de saúde competente é realizada pelos responsáveis por estabelecimentos públicos ou privados educacionais, de cuidado coletivo, de serviços de unidades laboratoriais, instituições de pesquisa e pelo cidadão (VIANA, FREITAS \& GIATTI, 2016).

No Brasil, a probabilidade de crianças menores de um ano serem hospitalizadas ou chegarem a óbito por doença diarreica aguda, nas microrregiões brasileiras, é maior nas regiões Norte e Nordeste. Essa doença diarreica tem raízes multifatoriais que poderiam ser evitadas se houvesse cuidados infantis adequados, acesso a serviços de saúde e boas condições de saneamento básico (BÜHLER et al., 2014; CABRAL, CUNHA \& CARDOSO, 2018).

Os usos mais frequentes da água que podem afetar a saúde do homem são classificados da seguinte forma: ingestão direta, preparação de alimentos, higiene pessoal e do ambiente, agricultura, processos industriais e atividades de lazer (RITÁ, SANTOS \& MORAIS, 2016). A qualidade da água ingerida depende da fonte, do controle e do tipo de processo de tratamento, desde sua origem, passando por diversas operações unitárias até alcançar a rede de distribuição, cujos parâmetros de controle quantitativo e qualitativo devem ser necessariamente monitorados, em condições potáveis, e, assim, evitar a propagação de doenças de transmissão hídrica (VON SPERLING, VERBYLA \& OLIVEIRA, 2020).

Um aspecto importante a ser considerado nesse contexto é se os indicadores operacionais de abastecimento de água refletem-se, com eficiência, sobre os indicadores de saúde pública. Isto é, se a melhoria dos serviços reduz a ocorrência de doenças. Portanto, a presente pesquisa teve como objetivo analisar indicadores operacionais de abastecimento de água relacionando-os com indicadores de saúde pública, visando seus impactos e sua eficiência no planejamento, na gestão e na política de prevenção de doenças pelos serviços de saneamento básico. Esse procedimento também permitiu comparar resultados com estudos similares realizados por outros autores da literatura (BARBOSA, LIMA \& BRUSCA, 2016; MAIELLO et al., 2015), preenchendo uma lacuna científica relevante do setor de saneamento básico no estado do Amapá e até mesmo na Amazônia.

A pesquisa testou as seguintes hipóteses:

- Há correlação entre os indicadores do Sistema Nacional de Informações sobre Saneamento (SNIS) e doenças de notificação compulsória de transmissão hídrica (ocorrência de doenças diarreicas agudas - ODDA), e essa correlação é inversamente proporcional. Isto é, quanto melhor são os indicadores do SNIS, menor serão as doenças (ODDA), porque ambos deveriam ser inversamente proporcionais, ou estar inversamente correlacionados com a melhoria dos indicadores operacionais da concessionária, a Companhia de Água e Esgoto do Amapá (CAESA);

- A melhoria dos indicadores operacionais resulta em menor exposição às doenças de veiculação hídrica nos diferentes municípios, indicando que a expansão dos serviços reduz proporcionalmente os potenciais agravos de saúde pública da população com o tempo. Na presente investigação, foram correlacionadas estatisticamente 20 variáveis operacionais de abastecimento de água. Essas variáveis foram pré-selecionadas com base em critérios, tais como existência, simultaneidade e consistência das séries temporais dos seis municípios e os indicadores de saúde pública realizados pela Superintendência de Vigilância em Saúde do Amapá (SVS-AP).

\section{METODOLOGIA}

\section{Área e período de estudo}

O estado do Amapá está localizado no extremo norte do Brasil e ocupa uma área territorial de $142.815 \mathrm{~km}^{2}$. Possui uma população estimada de 845.731 habitantes, distribuída em 16 municípios (IBGE, 2019). Atualmente, em alguns municípios do Amapá estão sendo realizados os Planos Municipais de Saneamento Básico - PMSB (UNIFAP, 2018), e esta pesquisa foi realizada com dados dos seguintes municípios: Amapá, Cutias, Itaubal, Laranjal do Jari, Mazagão e Vitória do Jari (Figura 1).

Na Tabela 1 constam as informações socioeconômicas de cada município e a quantidade de domicílios abastecidos por água pela concessionária local CAESA.

\section{Indicadores operacionais}

Neste estudo, foram levantados exclusivamente dados de indicadores dos municípios de Amapá, Cutias, Itaubal, Laranjal do Jari, Mazagão e Vitória do Jari, que constam oficialmente no desenvolvimento dos PMSB (UNIFAP, 2018). Para isso, foram pré-selecionados 20 indicadores operacionais de abastecimento de água considerando critérios de existência, simultaneidade e consistência de séries de dados temporais contidos no SNIS.

No SNIS, o eixo "água" apresenta 156 indicadores divididos em cinco grupos: informações gerais, financeiras, de qualidade, informações e indicadores de água, e indicadores operacionais (SNIS, 2019). Atualmente, as séries anuais estão disponibilizadas para o período entre 1996 e 2017, cujos 


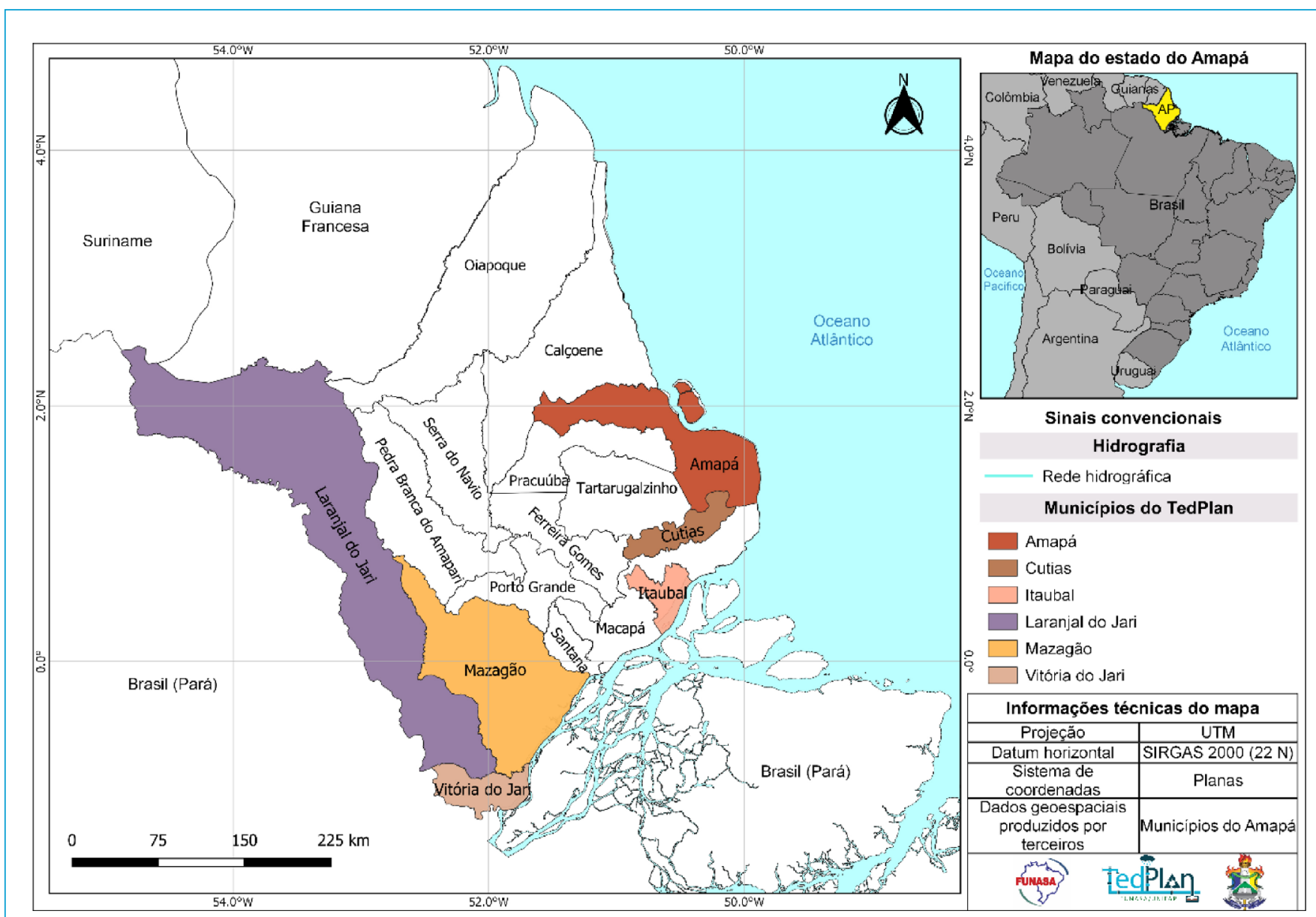

Fonte: adaptada pelos autores de acordo com a base cartográfica da Secretaria de Estado do Meio Ambiente (SEMA-AP) - UNIFAP (2018).

Figura 1 - Área de estudo com os municípios pesquisados.

Tabela 1 - Informações socioeconômicas dos seis municípios analisados.

\begin{tabular}{|c|c|c|c|c|c|c|}
\hline Municipios & $\begin{array}{l}\text { Area territorial } \\
\left(\mathrm{km}^{2}\right)(2018)\end{array}$ & $\begin{array}{c}\text { Densidade } \\
\text { demográfica } \\
(\text { hab./km²) (2010) }\end{array}$ & $\begin{array}{l}\text { Produto Interno } \\
\text { Bruto per capita } \\
\text { (R\$) (2017) }\end{array}$ & $\begin{array}{c}\text { Índice de } \\
\text { Desenvolvimento } \\
\text { Humano (2010) }\end{array}$ & $\begin{array}{c}\text { População } \\
\text { Estimada IBGE } \\
\text { (hab.) (2019) }\end{array}$ & $\begin{array}{l}\text { Sistema de Informação de } \\
\text { Atenção Básica (2013) }\end{array}$ \\
\hline Amapá & $8.454,85$ & 0,88 & $19.062,67$ & 0,642 & 9.109 & 790 \\
\hline Cutias & $2.179,11$ & 2,22 & $12.913,84$ & 0,628 & 5.983 & 559 \\
\hline Itaubal & $1.622,87$ & 2,50 & $12.713,02$ & 0,576 & 5.503 & 657 \\
\hline Laranjal do Jari & $30.782,99$ & 1,29 & $17.885,40$ & 0,665 & 50.410 & 5.114 \\
\hline Mazagão & $13.294,78$ & 1,53 & $12.003,39$ & 0,592 & 21.632 & 597 \\
\hline Vitória do Jari & $2.508,98$ & 5,01 & $17.885,40$ & 0,619 & 15.931 & 1.901 \\
\hline
\end{tabular}

Fonte: adaptada pelos autores de SIAB (2013) e IBGE (2019).

indicadores estão agrupados por eixo do saneamento básico classificados por famílias de informações.

Entretanto, por objetividade da análise, a pesquisa considerou 20 indicadores operacionais disponíveis no SNIS (Tabela 2). Foram considerados os indicadores que continham no mínimo $70 \%$ dos dados no período, evitando-se ausências significativas ou heterogeneidade de variâncias das séries históricas.

\section{Série temporal de doenças de} notificação compulsória de transmissão hídrica

No presente estudo, foi levantado o número de ocorrências de doenças de notificação compulsória de transmissão hídrica para o período entre 2007 e 2017 nos seis municípios. As doenças identificadas nessa categoria foram: cólera, febre tifoide, hepatite A, rotavírus e doenças diarreicas agudas. 
Tabela 2 - Indicadores operacionais de abastecimento de água com séries temporais válidas.

\begin{tabular}{|c|c|c|c|}
\hline Água total & Ligações de água / ligações faltantes & Perdas na distribuição & Volume de água \\
\hline $\begin{array}{l}\text { População total atendida com } \\
\text { abastecimento (hab.) }\end{array}$ & Quantidade de ligações ativas de água (lig.) & $\begin{array}{l}\text { Índice de perdas na } \\
\text { distribuição (\%) }\end{array}$ & Extensão da rede de água (km) \\
\hline $\begin{array}{l}\text { População urbana atendida com } \\
\text { abastecimento de água (hab.) }\end{array}$ & $\begin{array}{l}\text { Quantidade de economias ativas de água } \\
\text { (econ.) }\end{array}$ & \multirow{6}{*}{$\begin{array}{l}\text { Índice de perdas por } \\
\text { ligação (L.lig- }{ }^{-1} \text { dia--1) }\end{array}$} & Volume de água produzido (1000m³ dia-1) \\
\hline $\begin{array}{l}\text { Consumo médio per capita de água } \\
(\text { L. hab. dia-1) }\end{array}$ & $\begin{array}{l}\text { Quantidade de economias residenciais ativas } \\
\text { de água (econ.) }\end{array}$ & & Volume de água tratada em ETA $\left(1000 \mathrm{~m}^{3} \mathrm{dia}^{-1}\right)$ \\
\hline Índice de atendimento urbano de água (\%) & Quantidade de ligações totais de água (lig.) & & Volume de água consumido $\left(1000 \mathrm{~m}^{3} \mathrm{dia}^{-1}\right)$ \\
\hline Índice de consumo de água (\%) & \multirow{3}{*}{$\begin{array}{l}\text { Participação das economias residenciais de } \\
\text { água no total das economias de água (econ.) }\end{array}$} & & Volume de água faturado $\left(1000 \mathrm{~m}^{3} \mathrm{dia}^{-1}\right)$ \\
\hline Índice de atendimento total de água (\%) & & & \multirow{2}{*}{$\begin{array}{l}\text { Volume de água tratada por simples } \\
\text { desinfecção }\left(1000 \mathrm{~m}^{3} \text { dia- }^{-1}\right)\end{array}$} \\
\hline Índice de fluoretação de água (\%) & & & \\
\hline
\end{tabular}

Fonte: adaptada pelos autores de SNIS (2019)

A série de dados dessas doenças nos municípios foi disponibilizada pela SVS-AP em 2019. Um protocolo foi utilizado especificando quais doenças seriam necessárias para a pesquisa e qual a finalidade do levantamento. Os dados encontram-se disponíveis, exclusivamente, no Sistema de Informações de Agravos de Notificação (SINAN-NET), Unidade de Doenças Transmissíveis/Núcleo de Vigilância Epidemiológica (UDT/NVE) do Amapá.

\section{Análise estatística dos dados}

Os dados dos indicadores operacionais e de saúde foram tabulados e organizados para as análises estatísticas uni e multivariadas utilizando-se os softwares BioEstat 5.3 e R-Project, com nível de confiança de $95 \%(\alpha<0,05)$. Testes de Shapiro-Wilk foram aplicados para avaliar a normalidade, além de testes de inflação da variância (VIF) para evitar efeitos de colinearidade na análise de correlação (CRAWLEY, 2007).

Para a análise de correlação das variáveis, foram utilizados os métodos de análise de regressão simples e multivariada, com o suporte de uma Análise de Componentes Principais (PCA). A PCA teve como finalidade reduzir o número de variáveis colineares, eliminar sobreposições e definir formas representativas das combinações lineares das variáveis originais (CRAWLEY, 2007), além de complementar as análises de regressões. Assim, a PCA foi útil para identificar conjuntamente a relação entre características extraídas de dados (AYRES et al., 2007) quando vetores de características apresentam múltiplas dimensões (20 indicadores operacionais e doenças de NCTH).

O método de Friedman foi aplicado para testar múltiplas comparações de ODDA $(\alpha<5 \%)$ entre os diferentes municípios no período de estudo (20072017). Esse teste não utiliza os dados numéricos diretamente, e sim os postos ocupados por eles após a ordenação por grupo de análise separadamente. Após a ordenação, foi testada a hipótese de igualdade da soma dos postos de cada grupo (AYRES et al., 2007).

\section{RESULTADOS E DISCUSSÃO}

\section{Análise comparativa de ocorrências de doenças de notificação por município}

$\mathrm{Na}$ Tabela 3, apresenta-se a totalidade dos casos registrados de cólera, febre tifoide, hepatite A e rotavírus nos municípios estudados. Percebeu-se que em nenhum dos municípios houve ocorrência de cólera, sendo um aspecto positivo desse indicador. A cólera é uma doença bacteriana infecciosa intestinal aguda, transmitida por contaminação fecal-oral direta ou pela ingestão de água ou alimentos contaminados, relacionada com baixa cobertura de saneamento básico (REBELO, MAIO \& HOCHMAN, 2011).

O maior número de ocorrências de febre tifoide foi em Laranjal do Jari (23 casos notificados entre 2007-2017). A doença ocorreu também em Vitória do Jari (11 casos) e em Itaubal (um caso). Nos demais municípios, não houve registros dessa doença. A febre tifoide é uma doença transmissível associada às precárias condições sanitárias, à higiene pessoal e ambiental, ocorrendo frequentemente na forma de surtos relacionados com água e/ou alimentos contaminados (GUIMARÃES, MOMESSO \& PUPO, 2010). A doença persiste de forma endêmica no Brasil, com superposição de epidemias, especialmente nas regiões Norte e Nordeste (SANTOS \& ALCANTARA, 2014).

A maior ocorrência da hepatite A foi registrada em Mazagão (80 casos no período entre 2007-2017). Além desse registro, houve nove casos em Itaubal, sete em Laranjal do Jari, seis em Cutias, um no Amapá e nenhuma ocorrência em Vitória do Jari. A hepatite A é uma doença contagiosa, causada pelo vírus da Hepatite A (HAV), e também é conhecida como "hepatite infecciosa". Sua transmissão ocorre por via feco-oral, contato entre indivíduos e água ou alimentos contaminados pelo vírus (BARBOSA \& BARBOSA, 2013).

Neste estudo, notou-se uma baixa ocorrência do rotavírus nos municípios. Houve um caso em Itaubal e um em Mazagão, ocorridos no mesmo período. O rotavírus (rotavirose) é uma doença transmitida por via feco-oral, contato entre pessoas e ingestão de água e alimentos contaminados. Esses são um dos principais agentes virais causadores das doenças diarreicas agudas (TATE et al., 2012).

Em relação à ODDA da presente pesquisa, ao se comparar estatisticamente as medianas dos números de casos (teste de Friedman), o resultado foi próximo do significativo, no limite da significância $(\mathrm{p}=0,072)$. Nesse caso, é importante destacar que, pela falta de água tratada e pela inadequação operacional de sistemas de abastecimentos sem as devidas fases de desinfecção em alguns dos municípios (UNIFAP, 2018), o número de casos de diarreias agudas também tem sido frequentemente observado. Como essa doença de transmissão hídrica foi a de maior ocorrência nos municípios, foi realizado tratamento estatístico específico.

A Figura 2 mostra o percentual das ocorrências de doenças diarreicas agudas em Laranjal do Jari, que ficou acima de mil casos registrados ( $\approx 75 \%)$ entre os anos de 2007-2017, seguido do município de Vitória do Jari, com 800 casos 
Tabela 3 - Doenças de notificação compulsória de transmissão hídrica (2007-2017).

\begin{tabular}{|c|c|c|c|c|c|c|}
\hline Doença de Notificação Compulsória & Amapá & Cutias & Itaubal & Laranjal do Jari & Mazagão & Vitória do Jari \\
\hline Cólera & 0 & 0 & 0 & 0 & $\mathrm{O}$ & 0 \\
\hline Febre tifoide & 0 & $\mathrm{O}$ & 1 & 23 & $\mathrm{O}$ & 11 \\
\hline Hepatite A & 1 & 6 & 9 & 7 & 80 & $\mathrm{O}$ \\
\hline Rotavírus & 0 & $\mathrm{O}$ & 1 & $\mathrm{O}$ & 1 & 0 \\
\hline Doenças diarreicas agudas & 3.761 & 2.693 & 1.738 & 10.977 & 4.871 & 8.389 \\
\hline
\end{tabular}

Fonte: adaptada pelos autores de SVS-AP (2O2O).

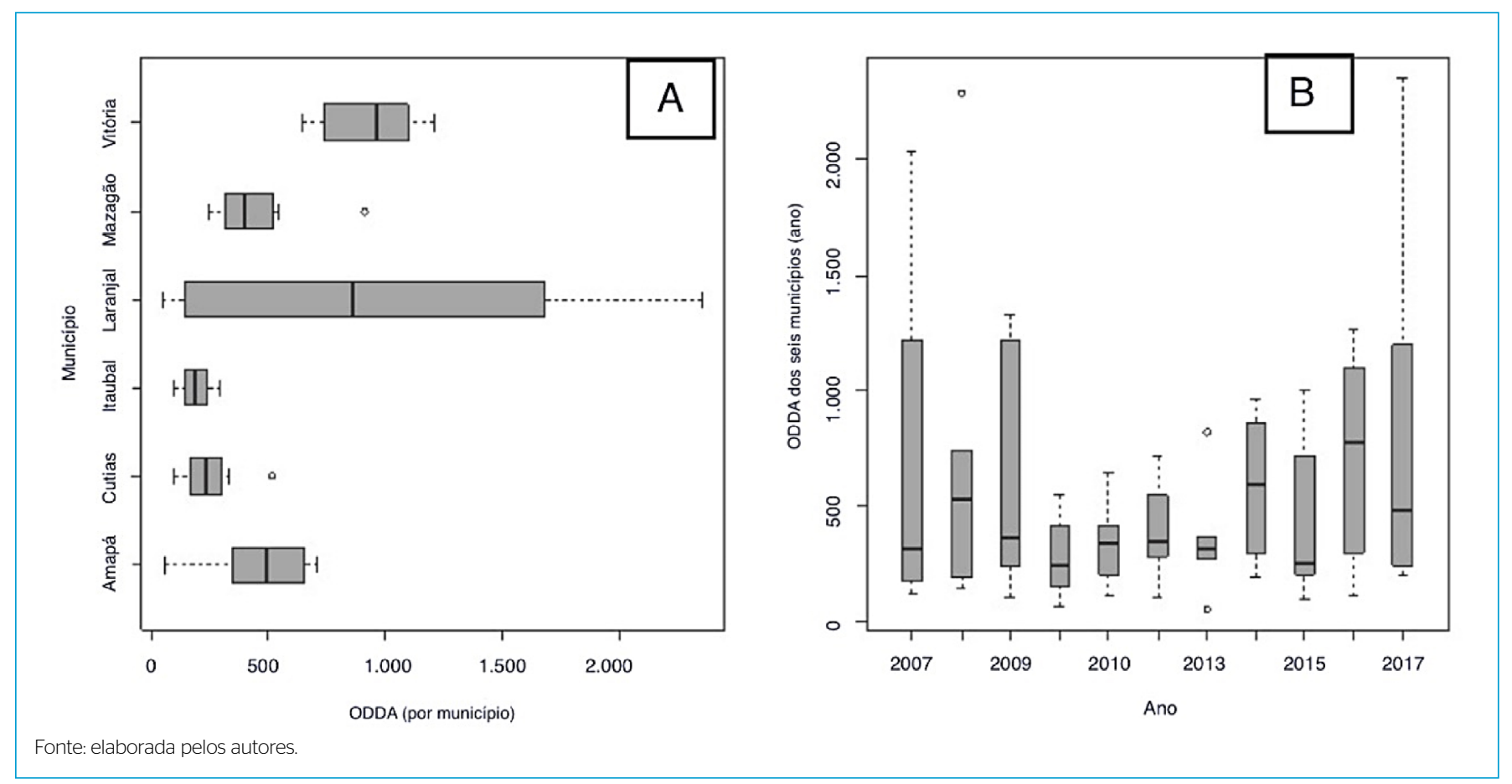

Figura 2 - (A) Variação das ocorrências de doenças diarreicas agudas (município); (B) evolução anual do número de ocorrências de doenças diarreicas agudas.

da doença $(\approx 65 \%)$. Houve uma concentração de valores de 2007 a 2015 em todos os municípios, sendo a menor concentração em Itaubal ( $\approx 2 \%)$ e a maior em Laranjal do Jari ( $\approx 75 \%$ ).

Essas ocorrências são registradas apenas quando o paciente, com sintomas da doença, realiza o diagnóstico em uma Unidade Básica de Saúde (UBS) do município. Uma vez confirmada, a doença é registrada no banco de dados da Vigilância em Saúde Municipal, passando tais informações para a Vigilância Estadual (SOUSA et al., 2012).

É importante compreender como as pessoas envolvidas percebem o risco associado com as doenças provenientes da água, a gravidade de muitas dessas doenças e suas motivações para adotar comportamentos preventivos. Essa compreensão é fundamental para que se possa prevenir a difusão de doenças hídricas (GUEDES et al., 2015).

$\mathrm{Na}$ Figura 3, indica-se, espacialmente, por meio de tons de cores e em escala temporal de dez anos (entre 2007-2017), as médias do número de ODDA por município. As cores mais escuras indicam maior número relativo de casos e as cores mais claras, o inverso. É importante frisar que a qualidade dos recursos hídricos tem impacto direto sobre a saúde dos indivíduos, que também está associada ao padrão de uso das águas. Se essas estiverem com a qualidade comprometida, podem causar efeitos deletérios sobre a saúde do usuário, particularmente quando as condições socioeconômicas e de infraestrutura não são favoráveis (MASUKAWA et al., 2016).

Para testar diferenças entre as medianas dos casos de diarreias entre os municípios, foi aplicado o teste não paramétrico Friedman $(\mathrm{p}<0,05)$. Todos os valores de $\mathrm{p}$ indicados por " $(\mathrm{p}<0,05)$ " são considerados significativos. Por exemplo, houve diferenças significativas de ODDA entre os municípios de Amapá e Cutias; Amapá e Itaubal; Amapá e Laranjal do Jari; e Amapá e Vitória do Jari $(\mathrm{p}<0,05)$ (retângulo destacado na Figura 4).

Na Figura 4, mostrou-se onde ocorreram diferenças significativas de ODDA nos municípios em função do tempo. Por exemplo, retirando-se da análise o município de Amapá (maior número de dados não disponíveis na série), Mazagão torna-se o município que mais se destacou em termos relativos ao indicador ODDA. Nesse caso, quando se aplica novamente o teste de Friedman, os municípios de Mazagão e Itaubal, e Mazagão e Laranjal do Jari também apresentaram diferenças significativas, com $\mathrm{p}<0,05$ (retângulo destacado na Figura 5).

Na Figura 5, identificam-se apenas duas barras significativas $(p<0,05)$, referentes a Itaubal e Mazagão, e Laranjal do Jari e Mazagão. Os demais municípios 


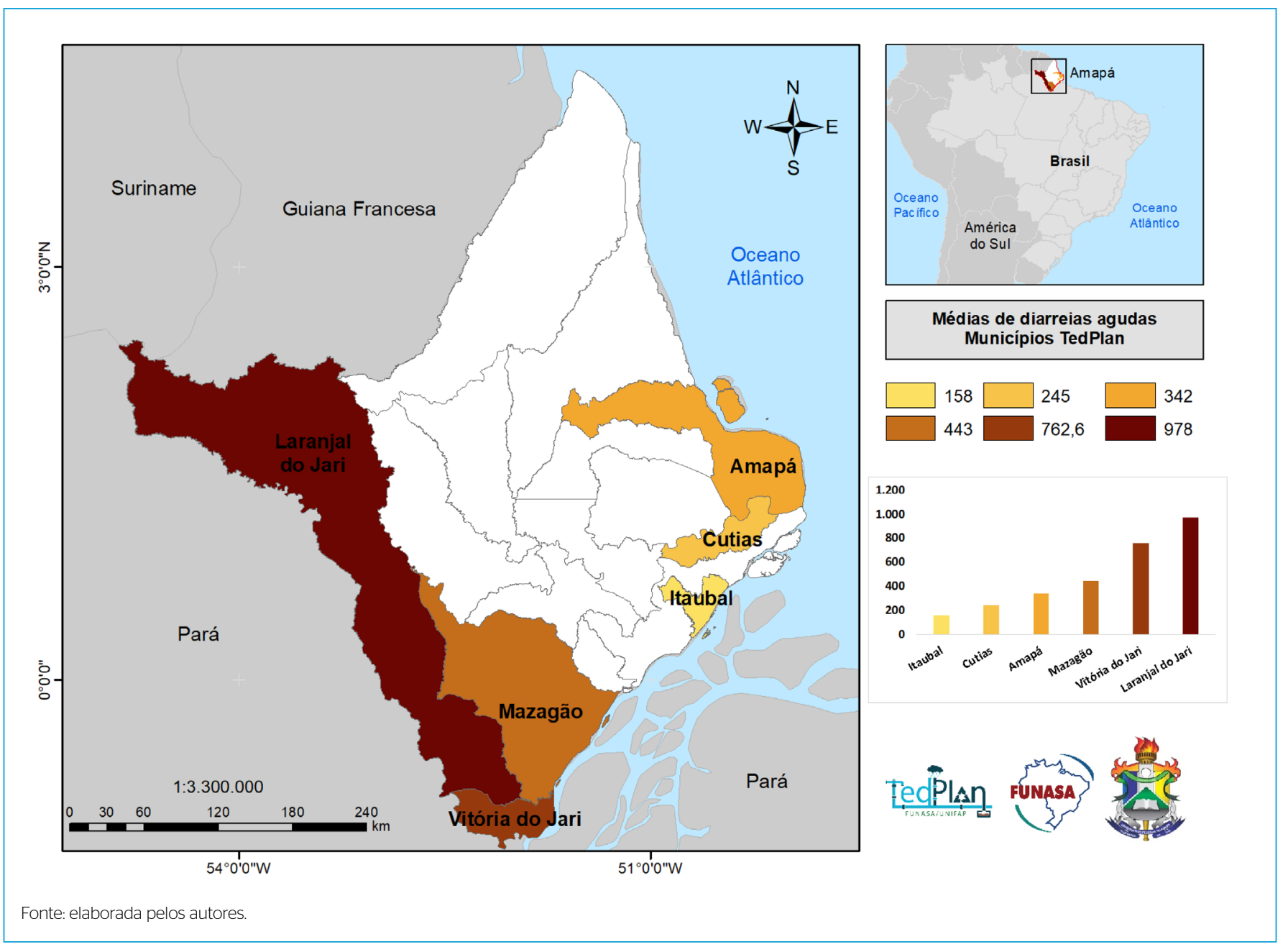

Figura 3 - Médias anuais de ocorrências de doenças diarreicas agudas por município.

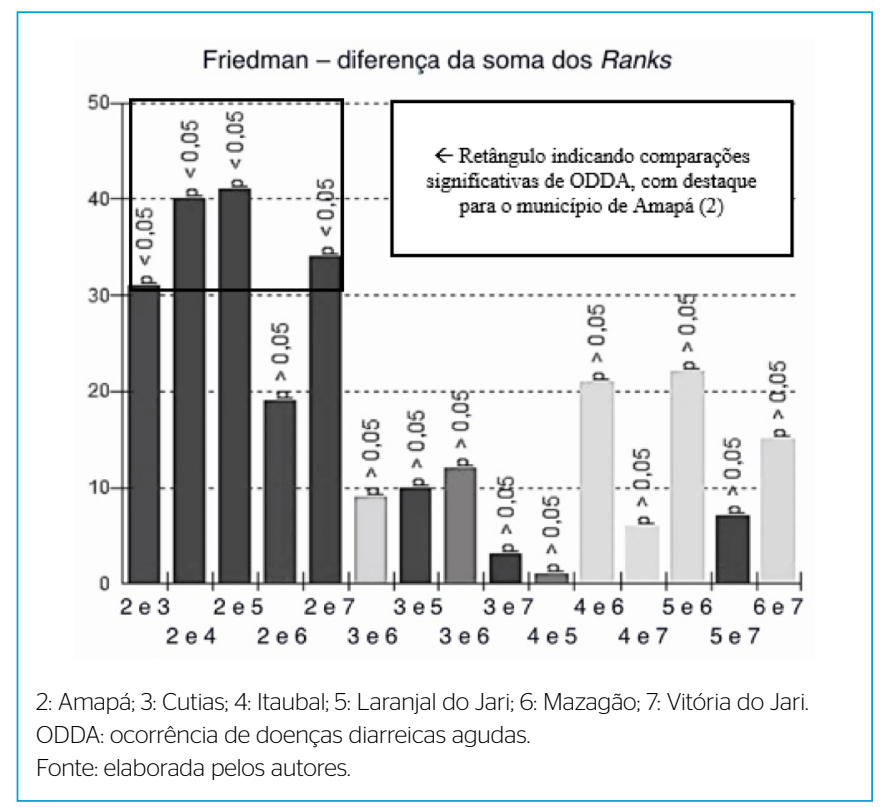

Figura 4 - Teste de Friedman para comparar ocorrências de doenças diarreicas agudas nos municípios (2007-2017).

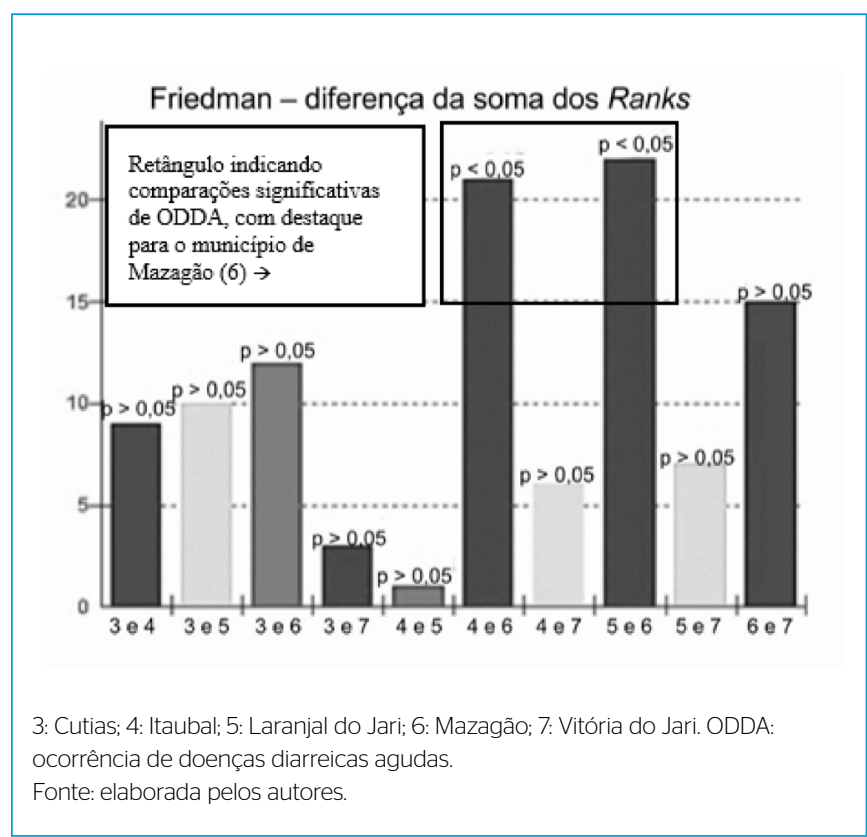

Figura 5 - Comparação dos casos de diarreias agudas entre os municípios. 
parecem não apresentar diferenças significativas entre si. No entanto, é importante ressaltar que, pelo menos em termos de ODDA, foi observado um relativo padrão de homogeneidade entre os municípios.

\section{Correlações dos indicadores de abastecimento de água (Sistema Nacional de Informações sobre Saneamento) com doenças (ocorrência de doenças diarreicas agudas) aplicando-se a Análise de Componentes Principais}

O número de ocorrências de diarreias agudas nos seis municípios variou positiva e significativamente $(\mathrm{p}<0,05)$ com as seguintes variáveis operacionais de abastecimento de água dos municípios: população total atendida com abastecimento (PTAA), quantidade de economias ativas de água (QEA), volume de água faturado (VAF), quantidade de economias residenciais ativas de água (QERA), quantidade de ligações totais de água (QLTA) e participação das economias residenciais de água no total das economias de água (PERA). Essas variáveis conjuntamente explicam aproximadamente $60 \%$ da variação total dos casos de ODDA, sabendo-se que os coeficientes foram positivos para PTAA, VAF e PERA, e negativos para QEA e QERA, não sendo significativo para QLTA (Tabela 4). Quando o coeficiente angular da variável específica é positivo, a ODDA tende a aumentar proporcionalmente, ocorrendo o inverso quando é negativo. Um mapa de PCA sintetiza essas variações entre variáveis interdependentes projetadas em dois eixos ( Dim $_{1}$ e Dim 2 ) (Figura 6).

A interpretação mais objetiva da PCA é que o eixo Dim $_{1}$ (horizontal) explica $45,10 \%$ da variação de todas as variáveis. Do mesmo modo, o eixo $\operatorname{Dim}_{2}$ (vertical) explica apenas $13,17 \%$ da variação conjunta das variáveis. O sentido das setas indica quão correlacionado estão as diversas variáveis do modelo em relação a ambos os eixos da PCA. Na Tabela 4 estão detalhadas individualmente cada regressão multivariada e a interpretação dessas correlações em relação às ODDA.

As regressões podem ser hierarquizadas do maior para o menor grau de importância (significância valor $\mathrm{p}$ ou explicabilidade $-\mathrm{R}_{\text {aj }}^{2}$ ) em relação aos indicadores operacionais por município e sua tendência no tempo: 1 - QEA; 2 - QLA; 3 - VAF; 4a - VAC e 4b - VAT; 5 - VAP; 6 - QLTA; e 7 - IAU.

- A análise e a interpretação dos indicadores relevantes indicaram quais variáveis foram significativamente mais influenciadas pela variável categórica "município" e em função da sua evolução no tempo:

- ODDA foi significativamente influenciada pelas variáveis independentes PTAA, VAF, QLTA e PERA (positivamente); e QEA e QERA (negativamente);

- Não houve influência significativa de ODDA quando se considerou apenas sua evolução no tempo ( $\mathrm{p}=0,73)$ e $\mathrm{R}_{- \text {-aj }}^{2}=29,8 \%$ apresentou explicabilidade para IAT, ano e municípios conjuntamente $(\mathrm{p}<0,001)$;

- As variações de ODDA em relação às variáveis independentes apresentaram resultados, às vezes conflitantes. Por exemplo, é inconsistente afirmar que quanto mais elevados forem os valores de PTAA, VAF e QLTA, maiores serão "também" os casos de doenças. Logo, esses resultados não eram esperados. Portanto, esperava-se uma redução temporal proporcional dos casos de ODDA em relação a essas variáveis.Além disso, se a QLTA aumentou, deveria haver redução de ODDA. Porém, ambos foram positivos e proporcionais. Isso significou que quanto maior for a QLTA nos seis municípios também seria maior a ODDA. Esta "incoerência" pode ter duas explicações:

- A série de dados dos indicadores pode ter consistência frágil.

Tabela 4 - Significância dos indicadores operacionais por município em função do tempo.

\begin{tabular}{|c|c|c|c|}
\hline Indicadores & Influência dos indicadores por município e tempo & $\begin{array}{l}\text { Evolução anual } \\
\qquad(p<0,05)\end{array}$ & $\mathrm{R}_{\text {aj }}^{2}$ \\
\hline QLA & *Laranjal do Jari $p=0,01$ e Vitória do Jari $p=0,03$ & $0,0002^{*}$ & 0,91 \\
\hline QEA & *Laranjal do Jari $p<0,001$, Mazagão $p=0,023$ e Vitória do Jari $p<0,001$ & $0,001^{*}$ & 0,93 \\
\hline QERA & NS (para nenhuma variável), exceto pelo tempo/ano $p=0,02$ & NS & 0,06 \\
\hline VAP & *Laranjal do Jari $p<0,001$ & $0,001^{*}$ & 0,75 \\
\hline VAC E VAT & ${ }^{*}$ Cutias $p=0,035$, Itaubal $p=0,02$ e Laranjal do Jari $p<0,001$ & $0,001^{*}$ & 0,81 \\
\hline VAF & *Laranjal do Jari $p<0,001$, Mazagão $p=0,038$ e Vitória do Jari $p=0,048$ & $0,001^{*}$ & 0,86 \\
\hline VATSD & ${ }^{*}$ Cutias $p=0,00021$, Itaubal $p=0,02$ e Laranjal $p=0,048$ & $0,0017^{*}$ & 0,19 \\
\hline QLTA & *Laranjal do Jari $p<0,0001$ e Vitória do Jari $p=0,00023$ e Laranjal do Jari $p=0,048$ & $0,0017^{*}$ & 0,74 \\
\hline CMPA & $\begin{aligned}{ }^{*} \text { Cutias } p=0,001 \text {, Itaubal } p=0,011 \text {, Laranjal do Jari } p=0,07 \text {. Próximo do limite da significância, Vitória do Jari } p=0,00023 \\
\text { Laranjal do Jari } p=0,048 \text { e Vitória do Jari } p=0,002\end{aligned}$ & $0,001^{*}$ & 0,18 \\
\hline IAU & ${ }^{*}$ Cutias $p<0,001$, Itaubal $p<0,001$, Laranjal do Jari $p<0,001$ e Vitória do Jari $p<0,0001$ & NS & 0,71 \\
\hline PERA & *Cutias, Itaubal, Mazagão, Laranjal do Jari e Vitória do Jari p < 0,001 & NS & 0,58 \\
\hline PTAA & *Laranjal do Jari e Vitória do Jari, todos p < 0,001 & $0,001^{*}$ & 0,81 \\
\hline IPL & ${ }^{*}$ Cutias $p<0,001$, Itaubal $p<0,001$, Laranjal do Jari $p<0,0011$ e Vitória do Jari $p=0,003$ & $0,001^{*}$ & 0,42 \\
\hline ICA & $\begin{array}{c}\text { *Todos os componentes independentes com a variável município ( } p<0,01) \text {. Cutias } p<0,001 \text {, Itaubal } p<0,0011 \\
\text { Laranjal do Jari } p<0,0011 \text { e Vitória do Jari } p<0,001\end{array}$ & $0,001^{*}$ & 0,51 \\
\hline IAT & *por todos os componentes independentes: variável categórica município ( $p<0,01)$, somente Laranjal do Jari $p<0,0004$ & NS & 0,11 \\
\hline
\end{tabular}

*significativo; QLA: quantidade de ligações ativas de água; QEA: quantidade de economias ativas de água; QERA: quantidade de economias residenciais ativas de água; VAP: volume de água produzido; VAC: volume de água consumido; VAT: volume de água tratada em Estações de Tratamento de Água; VAF: volume de água faturado; VATSD: volume de água tratada por simples desinfecção; QLTA: quantidade de ligações totais de água; CMPA: consumo médio per capita de água; IAU: índice de atendimento urbano de água; PERA: participação das economias residenciais de água no total das economias de água; PTAA: população total atendida com abastecimento; IPL: índice de perdas por ligação; ICA: índice de consumo de água; IAT: índice de atendimento total de água; NS: não significativo.

Fonte: elaborada pelos autores (2O2O). 


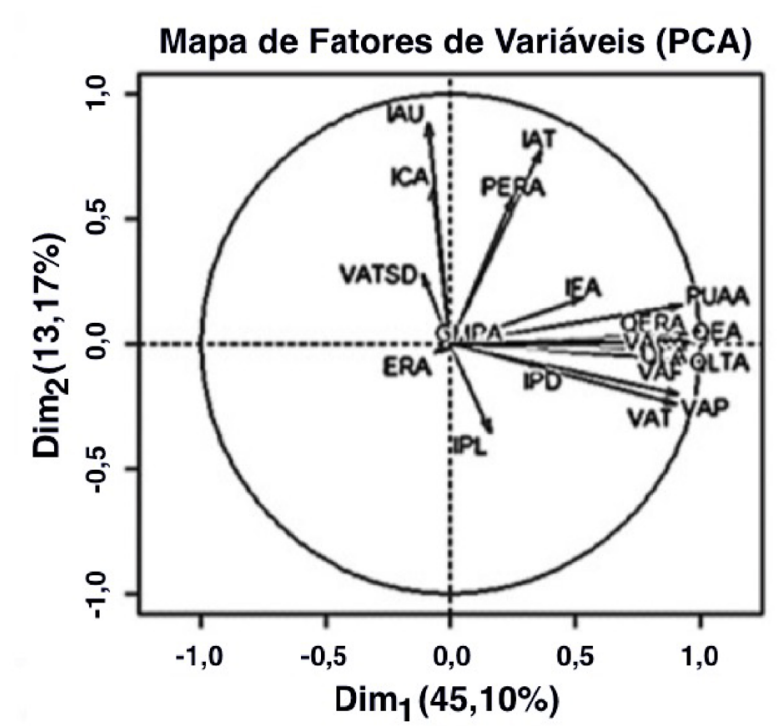

IAU: índice de atendimento urbano de água; ICA: índice de consumo de áqua; VATSD: volume de água tratada por simples desinfecção; ERA: extensão da rede de água; IPL: índice de perdas por ligação; IPD: índice de perdas na distribuição; VAT: volume de água tratada em Estações de Tratamento de Água - ETAs; VAP: volume de água produzido; VAF: volume de água faturado; QLTA: quantidade de ligações totais de água; QEA: quantidade de economias ativas de água; QERA: quantidade de economias residenciais ativas de água; PUAA: população urbana atendida com abastecimento de água; IFA: índice de fluoretação de água; PERA participação das economias residenciais de água no total das economias de água; IAT: índice de atendimento total de água; CMPA: consumo médio per capita de água

Fonte: elaborada pelos autores.

Figura 6 - Variação total dos indicadores operacionais nos eixos $\operatorname{Dim}_{1}$ e $\operatorname{Dim}_{2}$ (58,27\%).

- Há potencialmente subnotificações, tanto dos valores de ODDA quanto da influência de possíveis erros nos registros dos indicadores no SNIS.

- As regressões múltiplas (variáveis com alguns indicadores operacionais de abastecimento, principalmente "ano" e "município") foram mais eficientes para explicar as variações de ODDA do que quando submetidas às análises de regressões simples (com uma única variável explicativa).

As variáveis independentes (operacionais) que apresentaram correlação significativa com a ODDA são também as que melhor representaram os modelos regressivos da Tabela 4. Por exemplo, a variável VAF mostrou-se inconsistente por se apresentar positivamente correlacionada com a ODDA, quando se esperava um coeficiente negativo.

Portanto, a concepção dos indicadores deve contemplar as definições e a essência de cada parâmetro. Muitas variáveis utilizadas podem ser semelhantes, o que pode culminar na proposição de indicadores que expressam as mesmas características operacionais (FERRO et al., 2014), como mostrado na PCA (Figura 6). E alguns estudos revelam que um menor número de indicadores até pode facilitar a compreensão dos resultados. Assim, esses devem ser selecionados minuciosamente, a fim de que possam descrever, de maneira fidedigna, as variáveis avaliadas (SANTOS et al., 2018).

Todavia a avaliação de indicadores em relação ao porte do município e à concepção de minimização do número de indicadores, para explicação de determinado sistema de abastecimento de água, ainda é pouco explorada na literatura (HAMDAN, LIBÂNIO \& COSTA, 2019).

Portanto, a avaliação da eficiência das prestadoras dos sistemas de abastecimento de água deve se pautar em três premissas principais: a qualidade da água distribuída e os desempenhos operacional e financeiro. Caberá ao prestador de serviços de saneamento o fornecimento de água nos padrões de potabilidade vigentes e na quantidade necessária, além de dispor de capital suficiente para manutenção da qualidade do serviço (SCRIPTORE \& TONETO JÚNIOR, 2012).

Os resultados obtidos na presente análise mostram sinais evidentes de expansão do deficit dos serviços de abastecimento em relação à ODDA. Essas evidências estão refletidas na falta de controle e dificuldade da redução das ODDA nos municípios, indicando fragilidades estruturais, como mostram as regressões da Tabela 4 e as interpretações das Figuras de 2 a 6.

\section{CONCLUSÕES}

As hipóteses da pesquisa foram rejeitadas. Ou seja, a melhoria dos indicadores operacionais deveria reduzir proporcionalmente a ODDA no tempo em função da expansão e da melhoria do sistema de abastecimento de água da concessionária (CAESA). Pelo contrário, os impactos foram paradoxais.

A variável tempo (ano) não impactou significativamente a ODDA. Entretanto, houve significância quando todos os municípios são avaliados conjuntamente. Esse fato sugere que diferentes características dos sistemas operacionais de abastecimento de água tendem a apresentar diferentes impactos sobre a ODDA.

Os diferentes sistemas operacionais apresentaram tendência crescente de deficit dos serviços prestados no tempo. Porém, as inconsistências observadas podem ser explicadas por três fatores presentes na matriz de dados obtidos pelo SNIS e pela SVS:

- $\quad$ Falhas/erros no banco de dados do SNIS;

- Subnotificações dos dados da SVS; e

- A expansão dos sistemas de abastecimento não reflete em redução dos indicadores de ODDA, porque os indicadores operacionais (SNIS) também podem não ser consistentes.

Vale ressaltar que há algumas limitações das séries de dados do SNIS e da SVS-AP. Ainda são necessárias novas pesquisas para testar resultados futuros e confirmar se os atuais resultados representam uma tendência efetiva ou apenas uma exceção em relação aos indicadores avaliados.

\section{AGRADECIMENTOS}

Os autores agradecem o suporte técnico do Laboratório de Química, Saneamento e Modelagem de Sistemas Ambientais (LQSMSA) e o apoio financeiro do Conselho Nacional de Desenvolvimento Científico e Tecnológico (CNPq) \# 309684/2018-8, DPq/PROPESPg/UNIFAP e ao TEDPLAN/UNIFAP/FUNASA (TR-06/2018).

\section{CONTRIBUIÇÃO DOS AUTORES}

Araújo, E. P.: conceituação, curadoria de dados, metodologia, escrita - primeira redação, revisão e edição. Cunha, H. F. A.: conceituação, supervisão, escrita revisão e edição. Brito, A. U.: conceituação, escrita - revisão e edição. Cunha, A. C.: conceituação, análise estatística formal, obtenção de financiamento, metodologia, supervisão, escrita - revisão e edição. 


\section{REFERÊNCIAS}

AYRES, M.; AYRES JR, M.; AYRES, D. L.; SANTOS, A. A. S. BioEstat: Aplicações estatísticas nas áreas das ciências biológicas e médicas. Belém: Sociedade Civil Mamirauá; MCT-CNPq, 2007.

BARBOSA, A.; LIMA, S. C.; BRUSCA, I. Governance and efficiency in the Brazilian water utilities: a dynamic analysis in the process of universal access. Utilities Policy, v. 43, p. 82-96, 2016. http:// doi.org/10.1016/j. jun.2016.06.013

BARBOSA, D. A.; BARBOSA, A. M. F. Avaliação da completitude e consistência do banco de dados das hepatites virais no estado de Pernambuco, Brasil, no período de 2007 a 2010. Epidemiologia e Serviços de Saúde, v. 22, n. 1, p. 49-58, 2013. http://doi.org/10.5123/s1679-49742013000100005

BIASUTTI, S.; COELHO, E. R. C. Normatização de indicadores de perdas de água: a experiência das agências reguladoras no Brasil: a experiência das agências reguladoras no Brasil. Revista Dae, v. 67, n. 215, p. 17-24, 2019. http:// doi.org/10.4322/dae.2019.002

BRASIL. Lei $n^{\circ}$ 11.445, de 5 janeiro de 2007: Estabelece as Diretrizes Nacionais para o Saneamento Básico. Diário Oficial da União: seção 1, Brasília, DF, n. 5, p. 3, 8 jan. 2007.

BÜHLER, H. F.; IGNOTTI, E.; NEVES, S. M. A. D. S.; HACON, S. S. Análise espacial de indicadores integrados determinantes da mortalidade por diarreia aguda em crianças menores de 1 ano em regiões geográficas. Ciência \& Saúde Coletiva, v. 19, n. 19, p. 4131-4140, 2014. https://doi.org/10.1590/1413812320141910.09282014

CABRAL, A. A.; CUNHA, A. L., CARDOSO, M. D. T. Doenças Prevalentes na Infância: Diarreia e Desnutrição em uma Unidade de Saúde Bem Estruturada. Revista da Escola de Ciências Médicas de Volta Redonda, v. 1, n. 1, p. 1-9, 2018.

CRAWLEY, M. J. The R Book. 1. ed. Chichester, UK: Ed. John Wiley \& Sons Ltd, 2007. 951 p.

CUNHA, A. C. (Org.). Termo de Execução Descentralizada. Projeto: Planos Municipais de Saneamento Básico (PMSB) do Estado do Amapá. Macapá: UNIFAP/FUNASA, 2018. 52 p.

CUNHA, A. C.; CUNHA, H. F. A.; BRASIL JR, A. C. P.; DANIEL, L. A; SCHULZ, H. E. Qualidade microbiológica da água em rios de áreas urbanas e periurbanas no baixo Amazonas: o caso do Amapá. Engenharia Sanitária e Ambiental, v. 9. p. 322-328, 2004. http://doi.org/10.1590/S1413-41522004000400009

CUNHA, A. C.; NAZARE, A. S.; PANTOJA, S.; CUNHA, H. F. A.; SOUZA, J. Monitoramento de Águas Superficiais em Rios Estuarinos do Estado do Amapá sob Poluição Microbiológica. Boletim do Museu Paraense Emílio Goeldi Série Ciências Naturais, Belém, PA, v. 1, n. 1, p. 191-199, 2005.

FERRO, G.; LENTINI, E. J.; MERCADIER, A. C.; ROMERO, C. A. Efficiency in Brazil's water and sanitation sector and its relationship with regional provision, property and the independence of operators. Utilities Policy, v. 28, p. 42-51, 2014. http://doi.org/10.1016/j.jup.2013.12.001

GUEDES, G. R.; SIMÃO, A. B.; DIAS, C. A.; BRAGA, E. O. Risco de adoecimento por exposição às águas do Rio Doce: um estudo sobre a percepção da população de Tumiritinga, Minas Gerais, Brasil. Cadernos de Saúde Pública, v. 31, n. 6, p. 1257-1268, 2015. https://doi.org/10.1590/0102-311X00063514.
HAMDAN, O. H. C.; LIBÂNIO, M.; COSTA, V. A. F. Avaliação de indicadores aplicados a Sistemas de abastecimento de água de pequeno porte Engenharia Sanitária e Ambiental, v. 24, n. 6, p. 1183-1194, 2019. https://doi. org/10.1590/s1413-41522019185444

INSTITUTO BRASILEIRO DE GEOGRAFIA E ESTATISTICA (IBGE). IBGE cidades: Amapá, Cutias, Itaubal, Laranjal do Jari, Mazagão, Vitória do JariAmapá. 2019. Disponível em: https://cidades.ibge.gov.br/. Acesso em: O2 abr. 2020.

KERRY, J. H.; DAVID, W. H.; JOHN, C. C. Principles of Water Treatment. 1. ed. New Jersey, EUA: Ed. John Wiley \& Sons, INC, 2012. 654 p.

MAIELLO, A.; BRITTO, A. L. N. P.; MELLO, Y. R.; BARBOSA, P. S. O. (Un) used and (un)usable? The role of indicators in local decisionmaking. A Brazilian case study. Futures, n. 74, p. 80-92, 2015. https://doi. org/10.1016/j. futures.2014.11.002

MASUKAWA, M. L. T.; SOUZA, E. M.; GIMENES. E.; UCHIMURA, N. S. MORIWAKI, A. M.; UCHIMURA, T. T. Time series investigation of changes in seasonality of acute diarrhea hospitalizations before and after rotavirus vaccine in Southern Brazil. Cadernos de Saúde Pública, v. 32, n. 10, p. 1-10, 2016. https://doi.org/10.1590/0102-311X00080515

OLIVEIRA, E. D. C.; CASTELO-BRANCO, R.; SILVA, L.; SILVA, N.; AZEVEDO, J.; VASCONCELOS, V.; FAUSTINO, S.; CUNHA, A. First Detection of MicrocystinLR in the Amazon River at the Drinking Water Treatment Plant of the Municipality of Macapá, Brazil. Toxins, v. 11, n. 11, p. 1-21, 2019. http://doi. org/10.3390/toxins11110669

PAIVA, R. F. P. S.; SOUZA, M. F. P. de. Associação entre condições socioeconômicas, sanitárias e de atenção básica e a morbidade hospitalar por doenças de veiculação hídrica no Brasil. Cadernos de Saúde Pública, v. 34, n. 1, p. 40-50, 2018. http://doi.org/10.1590/0102-311×00017316

RASELLA, D. Impacto do Programa Água para Todos (PAT) sobre a morbimortalidade por diarreia em crianças do Estado da Bahia, Brasil. Cadernos de Saúde Pública, v. 1, n. 29, p. 40-50, 2013. Disponível em: https://www. scielosp.org/pdf/csp/2013.v29n1/40-50/pt. Acesso em: 13 out. 2021.

REBELO, F.; MAIO, M. C.; HOCHMAN, G. O princípio do fim: o "torna-viagem", a imigração e a saúde pública no Porto do Rio de Janeiro em tempos de Cólera. Estudos Históricos, v. 24, n. 47, p. 69-87, 2011. https://doi.org/10.1590/ S0103-21862011000100004

RITA, F. S.; SANTOS, C. S.; MORAIS, M. A. Doenças de Veiculação Hídrica: empoderamento para Educação em Saúde. In: XIII Congresso Nacional de Meio Ambiente de Poços de Caldas, Anais... Minas Gerais: Poços de Caldas, 2016, p. 1-8

SANTOS, V. F. N.; ALCANTARA, C. Incidência da febre tifoide e sua distribuição no Brasil - um estudo com base nos dados do cve/sinan dos anos de 2010 a 2013. Linkania Revista Cientifica, v. 1, n. 10, p. 1-12, 2014. Disponível em: http://linkania.org/master/article/view/263/178. Acesso em: 13 out. 2021.

SANTOS, R. C. L.; LIMA, A. S.; CAVALCANTI, E. B.; MELO, C. M.; MARQUES, M. N. Aplicação de índices para avaliação da qualidade da água da Bacia Costeira do Sapucaia em Sergipe. Engenharia Sanitária e Ambiental, v. 23, n. 1, p. 33-46, 2018. http://doi. org/10.1590/S1413-41522017159832 
SCRIPTORE, J. S.; TONETO JÚNIOR, R. A estrutura de provisão dos serviços de saneamento básico no Brasil: uma análise comparativa do desempenho dos provedores públicos e privados. Revista de Administração Pública, v. 46, n. 6. p. 1479-1504, 2012. http://doi.org/10.1590/S0034-76122012000600004

SUPERINTENDÊNCIA DE VIGILÂNCIA EM SAÚDE DO ESTADO DO AMAPÁ (SVS-AP). Boletins epidemiológicos. Governo do Estado do Amapá. 2020. Disponível em: https://svs.portal.ap.gov.br. Acesso em: 09 abr. 2020.

SISTEMA DE INFORMAÇÃO DE ATENÇÃO BÁSICA (SIAB). Departamento de Informática do SUS - DATASUS: Situação de Saneamento Básico nos Municípios de Amapá, Cutias, Itaubal, Laranjal do Jari, Mazagão e Vitória do Jari - Amapá. 2013. Disponível em: http://www2.datasus.gov.br/SIAB/index. php?area=01. Acesso em: 27 mar. 2020.

SISTEMA NACIONAL DE INFORMAÇÕES SOBRE SANEAMENTO (SNIS). Ministério das Cidades. Série Histórica: Amapá, Cutias, Itaubal, Laranjal do Jari, Mazagão, Vitória do Jari - Amapá. 2019. Disponível em: http://app4.mdr. gov.br/serieHistorica/. Acesso em: 09 abr. 2020.

SOUSA, S. P. O.; MASCARENHAS, M. D. M.; SILVA, M. C. B.; ALMEIDA, R. A. M. Conhecimento sobre doenças e agravos de notificação compulsória entre profissionais da Estratégia Saúde da Família no município de Teresina, estado do Piauí, Brasil - 2010. Epidemiologia e Serviços de Saúde, v. 21, n. 3, p. 465-474, 2012. http://doi.org/10.5123/s1679-49742012000300012

TATE, J. E.; BURTON, A. H; BOSCHI-PINTO, C.; STEELE, A. D.; DUQUE, J.; PARASHAR, U. D. Estimate of worldwide rotavirus-associated mortality in children younger than 5 years before the introduction of universal rotavirus vaccination programmes: a systematic review and meta-analysis. The Lancet Infectious Diseases, v. 12, n. 2, p. 136-141, 2012. http://doi.org/10.1016/ s1473-3099(11)70253-5

TSUTIYA, M. T. Abastecimento de água. 1. ed. São Paulo: Ed. Departamento de Engenharia Hidráulica e Saneamento da EPUSP; Politécnica da Universidade de São Paulo, 2006. 643 p.

VIANA, R. L.; FREITAS, C. M.; GIATTI, L. L. Saúde ambiental e desenvolvimento na Amazônia legal: indicadores socioeconômicos, ambientais e sanitários, desafios e perspectivas. Saúde e Sociedade, v. 25, n. 1, p. 233-246, 2016 http://doi.org/10.1590/S0104-12902016140843

VON SPERLING, M.; VERBYLA, M. E.; OLIVEIRA, S. M. A. C. Assessment of Treatment Plant Performance and Water Quality Data: A Guide for Students, Researchers and Practitioners. 1. ed. London, UK: Ed. IWA Publishing, 2020 $640 \mathrm{p}$. 